\title{
Understanding user requirements in context: a case study of developing a visualisation tool to map skills in an engineering organisation
}

\author{
Sian Joel-Edgar \\ Department of Computer Science \\ Bath University \\ Bath, U.K. \\ s.d.joel-edgar@bath.ac.uk
}

\author{
James Gopsill \\ Department of Mechanical Engineering \\ Bath University \\ Bath, U.K. \\ j.a.gopsill@bath.ac.uk
}

\begin{abstract}
We provide a case study of understanding the environment and work context of a visualisation tool used in a collaborative engineering organisation, in order to inform the design, development and evaluation of a knowledge management network tool. We utilise focus group and qualitative interview data to understand the dynamics of existing knowledge searches and the visual analytics process of collaborative working environments in an engineering domain. We discuss the enablers and functionality needed to create the network tool, and the envisaged challenges in its implementation. These challenges and enablers of knowledge management visualisation software are then discussed in relation to evaluating the tool in a way that is grounded in the contextual working environment in which it will be used.
\end{abstract}

Network visualisation; Knowledge management; social collaborative systems;

\section{INTRODUCTION}

It is widely recommended that requirements be derived from understanding the people and practices in which a software tool is being designed for [1]. For information and knowledge visualisation designers there is a need to understand the context in which the visualization tool will be used [2]. However in terms of understanding the development of information visualisation in a workplace [3, p7] it has been noted "hardly any papers devoted solely to analysis at this level [problem characterization] have been published". It has been maintained [4] that studying people and their task processes is still rarely carried out and there are only a few exceptions of academically published research. A first notable example was research [5] that sought to design information visualization tools for collaborative use and derived a framework that captured the analysis activities of co-located teams and individuals. Another example [6] conducted an ethnographic field study examining the ways in which building design teams used visual representations of data to coordinate their work. Furthermore there have been other contextual understanding of information visualisation in work practices but this has sought to evaluate software rather than to design software. For instance field observations [7], in which eight experts were observed in their workplace. Interviews [8], in which geo-visualisation experts were interviewed to learn about multi-disciplinary collaboration. As well as laboratory observations [9], whereby the ways in which people interacted with visualisations were studied. Additionally there have been others who have evaluated visualisations in context using quantitative methods (e. g. [10] and [11] etc).

The aim of this paper is to work towards a contextual understanding of the challenges and enablers of knowledge visualisation design in practice. Knowledge visualisations, in distinction to information visualisation, are the examination and use of visual representations to improve the transfer and creation of knowledge between at least two persons [12, 13 and 14]. We seek to understand the business context, social dynamics and work environments in which knowledge searching occurs and how a knowledge visualisation tool can exist within current organisational practices.

\section{KNOWLEDGE MANAGEMENT VISUALISATIONS IN ORGANISATIONS}

Knowledge Management has been defined as a "management perspective that offers theories strategies and methods to manage, i.e., to identify, access, share and create knowledge in organizations, with the aim to help an organization to compete by being more innovative, effective and thus more profitable.'[15 p239)]. It is a concept that stems from organisational learning [16, 17 and 18], and sees knowledge as a key productive and strategic resource in an organisation $[19,20,21,22]$. Although there are many diverging perspectives of knowledge, knowledge management can be seen as having three main objectives: 1) optimizing processes 2) introduction of systems for storing, identifying, retrieving, and gaining access to information 3) development of a corporate knowledge culture. It can also be categorised as containing four main processes [23]: 1) creation 2) the storage and retrieval 3) transfer and 4) the application of knowledge. In this paper we consider knowledge management visualisations within an engineering organisation. The visualisation tool we discuss aims to address the intentions of knowledge management, and specifically at the retrieval of information (the second knowledge management process - [23]).

In most knowledge related activities, the knowing of what others know is a necessary component for coordinated action to take place (e.g., [24]). As Busby stated, "learning from experience, and attending to the consequences of one's 
work, are strong norms in design organisations" [25, p103]. This means that effective knowledge creation and sharing require that the points of view of others be realistically imagined [26]. Therefore individuals working for an organisation need to communicate with each other by a number of different means, such as face-to-face conversations, telephone, electronic mail, ordinary mail, etc.

The performance of an organisation can be improved by helping workers learn more about one another so that they can make better use of the company's' human resources [27 and 28]. Among these resources, the work related knowledge of team members, often referred to as the team's intellectual capital or knowledge assets, is important.

A typical situation has been that the stored knowledge is retrievable only when an individual knows what it is and where it is. In the search for a solution to a problem, for example, an individual team member may know what the problem is but not know the possible solution. The successful retrieval of the solution thus requires the prior knowledge of what might be the solution and where the solution might be. This means that knowing where a possible solution is to be found can be more important than merely knowing the solution [29].

However, the amount of data organisations has been exploding, beyond the capability of human cognitive processes. Analyzing large data sets - so-called big datahas become a key basis of competition, underpinning new waves of productivity growth, innovation, and consumer surplus [30]. Analysis results of big data give meaningful quantitative base for further qualitative understanding for transferable meaningful knowledge.

According to boyd and Crawford [31] big data is, in many ways, a poor term. Manovich [32] observes, it has been used in the sciences to refer to data sets large enough to require supercomputers, although now vast sets of data can be analyzed on desktop computers with standard software. Big data is notable not because of its size, but because of its relationality to other data. Due to efforts to mine and aggregate data, big data is fundamentally networked. Its value comes from the patterns that can be derived by making connections between pieces of data, about an individual, about individuals in relation to others, about groups of people, or simply about the structure of information itself [31]. Therefore, we see that this would be an important new area to explore in connection to network relation and knowledge management in organisations.

\section{THE CASE STUDY}

In this paper we introduce a case of an engineering Catapult organisation within the U.K. The Catapult in questions is a collaborative engineering workplace that contains several technology companies, research and development laboratories, design and engineering departments, and joint research facilities with universities. The Catapult provided us as researchers a textbook example of a collaborative engineering workplace with its advantages and with its challenges in co-creation and collaborative engineering work.

In order to understand the contextual requirements of the network knowledge tool, seven formal documented interviews were carried out, recorded and transcribed. Additionally there were many informal meetings and discussions in which notes were taken. Finally a group meeting with strategic staff members (including knowledge management officers) was conducted in which notes were taken by two researchers and compared and analysed after the event. The formal interviews, in general, lasted approximately an hour in length, and provided an understanding of how knowledge is gathered currently within the Catapult, the social dynamics of searching for knowledge in a large organisation and the overall requirements for the tool. A framework for the interview questions was based on questions for understanding environments and work practices [4]. The interviews were then coded in Nvivo using a grounded theory [33] approach to elicit themes and generate hypothesis.

The interviewees described how and why individuals sought knowledge and people with skills. Scenarios that were envisaged in the interviews included: firstly a new staff member wanting to know about an area they have been tasked with, a staff member who has been with the organisation for some time, but wishes to know about a new subject area. The executive team who wish to understand the landscape of the organisation and where skills are lacking or abundant, or how the skill set of the organisation is changing over time (what are the new skills emerging or what are the organisations core skills). Finally the allocation of people to projects based on their knowledge of certain skills. This scenario is described in more detail in table 1 .

TABLE I. EXAMPLE USE SCENARIO

\begin{tabular}{|l|l|}
\hline Goal & $\begin{array}{l}\text { To identify the internal capabilities within the } \\
\text { Catapult. }\end{array}$ \\
\hline $\begin{array}{l}\text { Sub- } \\
\text { goals }\end{array}$ & $\begin{array}{r}\text { To show clusters of capabilities. } \\
\text { To search for potential capabilities } \\
\text { within the Catapult. } \\
\text { To show dominant capabilities. } \\
\text { To show where capabilities are lacking } \\
\text { in the organisation. }\end{array}$ \\
\hline $\begin{array}{l}\text { Main } \\
\text { actors }\end{array}$ & $\begin{array}{l}\text { Engineering Capability Managers (ECMs). } \\
\text { Pre- } \\
\text { conditi } \\
\text { ons }\end{array}$ \\
$\begin{array}{l}\text { A new project or idea has been put forward. } \\
\text { Resources need to be allocated, particularly for } \\
\text { Category } 3 \text { and } 4 \text { projects where risks are higher } \\
\text { (greater costs/resources and less precedence than } \\
\text { Category } 1 \text { or } 2 \text { projects). }\end{array}$ \\
\hline
\end{tabular}




\section{A. Description of the software}

Prototype network visualization was developed to address the use-case scenarios outlined in the interviews. Data was taken from the organisations' document corpus of completed project reports (that do not contain sensitive information which cannot be shared). Using this data, an algorithm was created which pulls topic themes and associated individuals (as described in the project reports). This was achieved by extracting all non-stop words from the reports, (that appeared regularly as there was a minimum occurrence threshold set). Clustering analysis was carried out to identify bi-grams and tri-grams of words and to further allow the topics to be grouped and for themes (skills) to emerge. This data was converted into a graphical file for conversion into a web based (NetworkX) tool. The network visualisation then showed the clustered terms, which reports the terms featured in and who were the authors of the reports (fig 1). This allowed the user to ascertain who has a skill within the organisation because of their contribution to a report that includes reference to that skill. This visualisation prototype was introduced into the case study site for a first testing phase in a social group environment (coffee room) in which engineers are asked to 'play' with the tool as well as answer some usability questions around core functionality.

A focus group was conducted to test the prototype. This consisted of 5 people, over an hour-long session, who were asked to pretend to act out the four scenarios outlined from the interviews. The focus group aimed to discover if the participants would gain insights from the visualization tool. The questioning framework [37] sought to ask the participants to formulate questions about the data that they expected the visualization tool to answer.

Figure 1: Screen shot of network visualisation (dark blue node=topics, light blue node $=$ report. and green node $=$ anonymised person

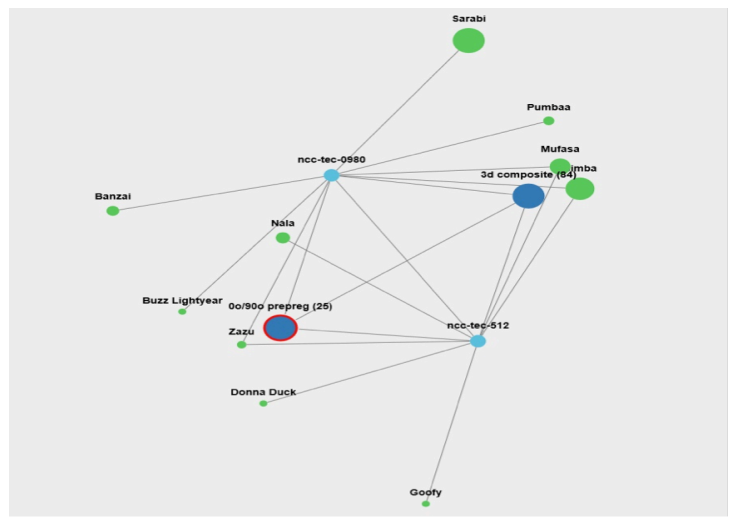

\section{B. Dynamics of accessing knowledge digitally}

In this paper we focus on the qualitative requirements of the data, which would be needed. In order to be able to collect a large amount of data (often called big data) from different organizations, the data would need to have certain features. Secondly in order to share this data there are challenges exhibited from the different values and cultures of collaborating companies.

The Catapult centres provide businesses access to manufacturing talent and facilities, with its aim being to rapidly allow prototypes to be tested and brought into the commercial domain whilst also allowing for scientific expertise to be accessed. However as the Catapult has grown it have become difficult to know the capabilities of the whole organisation and its staff. This has resulted in a knowledge-sharing problem.

Currently data, in which knowledge resides, can be found in various forms in the Catapult but is perceived to be found on their intranet. However strategies to access data usually involved web searches, extractions of academic papers and then accessing people via email prior to accessing internal knowledge sources. The interview data suggests people tend to carry out wide knowledge searches (utilising google etc), then people with specific knowledge before turning to internal documentation. However to comprehend the internal documentation also requires 'pointers' by key individuals that are known to them. It was generally felt that the internal documentation system was not good enough to search for skills and topics within the organization and supporting knowledge in the Catapult through the use of technology was not straightforward. A summary of the emerging themes found from the interview data were:

- Searching for knowledge through internal technical means is a low priority choice. People preferred to talk to knowledgeable people face-to-face.

- Persons are sought for 'lessons learnt' type knowledge.

- New starters may traverse the network differently unless guided (e.g. by knowledgeable people who sit near them, or fortuitous contacts).

- The importance of access to knowing who to contact (what they look like, who they work with, where they sit).

- High level (skimming) processes are carried out to scan documents (save time).

- There are bureaucratic hold ups to implementing knowledge management in socially complex environments.

- Confidentiality a major hurdle for accessing knowledge.

The inter-relationship between companies at the Catapult are complex, with influence, interpretations, and the needs of individuals often competing or generating conflicting accounts. This makes the task of understanding a network of 
knowledge within the Catapult all the more difficult. It also makes the development of software for that task equally as problematic. This is compounded if the engineering activity does not occur through a technological medium and knowledge is gained tacitly. Searl [34, p4] noted, "complex social structure of reality is, so to speak, weightless and invisible". This requires a software solution that maps people and associated knowledge that may be compartmentalised into knowledge areas that may shift over time and whose capabilities may not be formally documented. The attribution of people to capability may also not be formatted in a meaningful way to access implicit knowledge. The prototype software is based upon individuals and their associated knowledge explicitly referenced in project report documentation. Tacit knowledge would need to be elicited by liaising with staffing (based initially on their explicit knowledge as documented in project reports). However there are particular issues for new starters and how they approach searching for knowledge and where to start the search. Even persons who have been in the organisation for a while struggles to know the best avenue to take, especially if (key) people leave, there is a significant change in a person's skill set and if people move from where they usually sit.

\section{Knowledge sharing.}

In the Catapult there are many challenges to sharing knowledge and learning. Firstly, issues relating to information sharing and its growth in size. In large communities no-one can know the whole organisation. Large collaborations require some kind of division of labour, and that people perform their role at a specific time. In contrast an individual could not achieve large undertakings alone, group creativity enables a project to be divided and allocated to individuals with specific skills to meet the task at hand. To a certain extent this kind of set-up can be achieved in formal collaborative teams, with people working for the same company. However with organisations like the Catapult, "fields" of engineering expertise exist within a complex network of collaborating companies that are working within the umbrella organisation of the Catapult.

Furthermore as the Catapult has grown it has becomes increasingly more difficult to access knowledge. In particular, who has the knowledge in the organisation, and who is aware of that knowledge. Managers and engineers are unaware of what other engineers are doing. Managers in Catapults may not be aware of where skills lie in the wider organisation, where capabilities are missing or interrelate to each other. This requires the automatic extraction of data from sources that gives an indication of where knowledge lies, without a human gatekeeper knowing where capabilities lie and acting upon that knowledge to fulfil project demands.
Consequently people tend to work in silos, working in day-to-day teams of a size that are manageable to their knowledge base. The challenge in the Catapult is how to break boundaries between these silos. In the Catapult, these silos can form around distinct companies that are part of its organisation. However lessons can be learnt from knowledge in each distinct company, even if that knowledge may be company or product specific (assuming there are no security issues in sharing certain company specific knowledge).

\section{CONCLUSION}

Collaborative engineering environment such as the Catapult aim to gather, leverage and analyse their internal data to enable insights that will help their business practice [35] Business intelligence and analytics, such as the internal reporting strategy and its associated knowledge at the Catapult, allows for the transformation of the data into a visualisation and to draw data-driven insights [36]. However, many business intelligence and visualisation tools do not focus on the context in which they being used. It has already been maintained [4] in information visualization research studying people and their task processes are rarely carried out, except for a few exceptions [e. g., 5 and 6]. Consequently, this paper aims to outline the context of a knowledge management visualisation tool used in a Catapult. The needs, requirements and functionality of the knowledge network tool are provided in this paper, as are the perceived challenges to its implementation. This understanding is grounded within the working environment and specific context of the Catapult. We discuss how these are implemented into the software tool, and propose how these features of the software are evaluated.

\section{REFERENCES}

1. Shneiderman, B., \& Plaisant, C. (1987). Designing the user interface: Strategies for effective human-computer interaction. ACM SIGBIO Newsletter, 9(1), 6.

2. Plaisant, C. (2004, May). The challenge of information visualization evaluation. In Proceedings of the working conference on Advanced visual interfaces (pp. 109-116). ACM.

3. Munzner, T. (2009). A nested model for visualization design and validation. IEEE transactions on visualization and computer graphics, 15(6).

4. Lam, H., Bertini, E., Isenberg, P., Plaisant, C., \& Carpendale, S. (2012). Empirical studies in information visualization: Seven scenarios. IEEE Transactions on Visualization and Computer Graphics, 18(9), 1520-1536.

5. Isenberg, P., Zuk, T., Collins, C., \& Carpendale, S. (2008, April). Grounded evaluation of information visualizations. In Proceedings of the 2008 Workshop on BEyond time and errors: novel evaLuation methods for Information Visualization (p. 6). ACM.

6. Tory, M., \& Staub-French, S. (2008, April). Qualitative analysis of visualization: A building design field study. In Proceedings of the 2008 Workshop on BEyond time and 
errors: novel evaLuation methods for Information Visualization (p. 7). ACM.

7. Sedlmair, M., Baur, D., Boring, S., Isenberg, P., Jurmu, M., \& Butz, A. (2008). Requirements for a mde system to support collaborative in-car communication diagnostics. In CSCW Workshop on Beyond the Laboratory: Supporting Authentic Collaboration with Multiple Displays.

8. Brewer, I., MacEachren, A. M., Abdo, H., Gundrum, J., \& Otto, G. (2000). Collaborative geographic visualization: Enabling shared understanding of environmental processes. In Information Visualization, 2000. InfoVis 2000. IEEE Symposium on (pp. 137-141). IEEE.

9. Robinson, A. C. (2008, October). Collaborative synthesis of visual analytic results. In Visual Analytics Science and Technology, 2008. VAST'08. IEEE Symposium on (pp. 67-74). IEEE.

10. Kobsa, A. (2004, October). User experiments with tree visualization systems. In Information Visualization, 2004. INFOVIS 2004. IEEE Symposium on (pp. 9-16). IEEE.

11. Willett, W., Heer, J., \& Agrawala, M. (2007). Scented widgets: Improving navigation cues with embedded visualizations. IEEE Transactions on Visualization and Computer Graphics, 13(6), 1129-1136.

12. Burkhard, R. A. (2004, July). Learning from architects: the difference between knowledge visualization and information visualization. In Information Visualisation, 2004. IV 2004. Proceedings. Eighth International Conference on (pp. 519524). IEEE.

13. Burkhard, R., \& Meier, M. (2004, June). Tube map: Evaluation of a visual metaphor for interfunctional communication of complex projects. In Proceedings of $I$ Know (Vol. 4, pp. 449-456).

14. Eppler, M. J., \& Burkhard, R. A. (2007). Visual representations in knowledge management: framework and cases. Journal of knowledge management, 11(4), 112-122.

15. Burkhard, R. A. (2005). Impulse: Using knowledge visualization in business process oriented knowledge infrastructures. Journal of Universal Knowledge Management, 2, 170-188.

16. Argyris, C., \& Schön, D. A. (1978). A theory of action perspective. Addison-Wesley Publishing Company.

17. Fiol, C. M., \& Lyles, M. A. (1985). Organizational learning. Academy of management review, 10(4), 803-813.

18. Senge, P. (1990). The fifth discipline: The art and science of the learning organization. New York: Currency Doubleday.

19. Grant, R. M. (1996). Prospering in dynamically-competitive environments: Organizational capability as knowledge integration. Organization science, 7(4), 375-387.

20. Nonaka, I. (1991). Models of knowledge management in the West and Japan.

21. Nonaka, I., \& Takeuchi, H. (1995). The knowledge creation company: how Japanese companies create the dynamics of innovation. New York.

22. Spender, J. C. (1996). Making knowledge the basis of a dynamic theory of the firm. Strategic management journal, 17(S2), 45-62.

23. Alavi, M., \& Leidner, D. E. (2001). Research commentary: Technology-mediated learning-A call for greater depth and breadth of research. Information systems research, 12(1), 110 .
24. Krauss, R.M. and Fussell, S.R., (1991). Perspective-taking in communication representation of others' knowledge in reference. Social Cognition, 9(1), pp.2-24.

25. Busby, J., S., (1998) The neglect of feedback in engineering design organisations. Design Studies, Vol(19), Issue 1, January, pp.103-117.

26. Brown, R., (1981). Social Psychology. New York: The Free Press

27. Moreland, R.L., (1999). Transactive memory: Learning who knows what in work groups and organizations. In L.L. Thompson, J.M Levine and D.M. Messick, eds. Shared Cognitions in Organizations: The Management of Knowledge. Mahwah: Erlbaum, pp.3-31.

28. Lindkvist, L., (2005). Knowledge communities and knowledge collectivities: A typology of knowledge work in groups. Journal of Management Studies, 42(6), pp.1189-210.

29. Koskinen, K.U. and Aramo-Immonen, H. (2008) Remembering with help of personal notes in project work context, International Journal of Managing Projects in Business, Vol 1, No 2, 2008, pp. 193-205.

30. McKensey (2011) Manyika et al. Big data: The next frontier for innovation, competition, andproductivity.http://www.mckinsey.com/insights/business_t echnology/big data_the_next_frontier_for_innovation >.[21.2.2014].

31. Boyd, D., \& Crawford, K. (2011, September). Six provocations for big data. In $A$ decade in internet time: Symposium on the dynamics of the internet and society (Vol. 21). Oxford: Oxford Internet Institute.

32. Manovich, L. (2011) 'Trending: The Promises and the Challenges of Big Social Data', Debates in the Digital Humanities, ed M.K.Gold. The University of Minnesota Press, Minneapolis,

33. Strauss, Anselm, and Juliet Corbin. "Grounded theory methodology." Handbook of qualitative research 17 (1994): 273-85.

34. Searle, J. R (1995) The Construction of Social Reality, New York: The Free Press, 1995

35. Watson, H. J., \& Wixom, B. H. (2007). The current state of business intelligence. Computer, 40(9).

36. Lim, E., Chen, H., and Chen, G. (2013). Business Intelligence and analytics: Research directions. Trans. in Information Systems, ACM Press, 3,4

37. Saraiya, P., North, C., \& Duca, K. (2005). An insight-based methodology for evaluating bioinformatics visualizations. IEEE transactions on visualization and computer graphics, 11(4), 443-456

\section{AUTHORS' BACKGROUND}

\begin{tabular}{|c|c|c|c|}
\hline $\begin{array}{l}\text { Your } \\
\text { Name }\end{array}$ & * Title & $\begin{array}{l}\text { Research } \\
\text { Field }\end{array}$ & $\begin{array}{l}\text { Personal } \\
\text { website }\end{array}$ \\
\hline $\begin{array}{l}\text { Sian Joel- } \\
\text { Edgar }\end{array}$ & $\mathrm{Dr}$ & $\begin{array}{l}\text { Computer } \\
\text { Science }\end{array}$ & \\
\hline \begin{tabular}{|l|} 
James \\
Gopsill
\end{tabular} & $\overline{\mathrm{Dr}}$ & $\begin{array}{l}\text { Mechanical } \\
\text { Engineering }\end{array}$ & \\
\hline
\end{tabular}

